\title{
Silicon Surface Modification with Supported Phospholipids Bilayer for Biosensor based on Imaging Ellipsometry
}

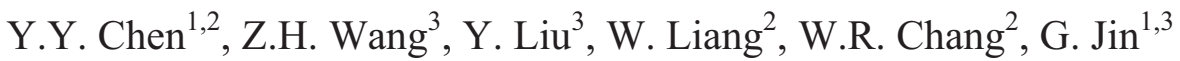 \\ ${ }^{1}$ Institute of Nano-tech and Nano-bionics, Chinese Academy of Sciences, Suzhou 215125, P.R. China \\ ${ }^{2}$ Institute of Biophysics, Chinese Academy of Sciences, Beijing 100101, P.R. China \\ ${ }^{3}$ Institute of Mechanics, Chinese Academy of Sciences, Beijing 100080, P.R. China
}

\begin{abstract}
A method is presented for fabricating supported phospholipids bilayer on silicon surface for biosensor based on imaging ellipsometry. The lipid bilayer prepared by covalent immobilization of phosphatidylethanolamine layer on silicon surface followed by adsorption of PEG functionalized phospholipids layer, is highly protein resistant and stable, which can withstand multiple cycles of drying and rehydration, remaining intact in various buffers. Proteins covalently immobilized on the bilayer by the terminal pnitrophenylcarbonyl or carboxyl group of PEG remain higher bioactivity than that immobilized on silicon surface directly.
\end{abstract}

Keywords - imaging ellipsometry, phospholipids-supported bilayer, biosensor, microfluidic array.

\section{INTRODUCTION}

The concept of biosensor based on imaging ellipsometry is reported in 1995 [1]. As the visualization method of biosensor, imaging ellipsometry which combines the power of ellipsometry with microscopy [2] provides a larger field of view for the biosensor (several square centimeters) with a high spatial resolution in the order of micron (laterally) and sub-nanometer (vertically), which makes the biosensor convenient to detect multiple analytes in a microarray simultaneously. Another interesting feature of the biosensor is label free for biomolecule detection, which avoids problems caused by the use of labeling like radioactive isotopes or fluorophores [3]. However, one drawback of the biosensor is that it can not distinguish specific binding and non-specific adsorption. It is necessary to develop a special surface modification to minimize the non-specific binding.

Supported phospholipids bilayer (SPB) at interfaces have actually been shown a good validity in preventing protein adsorption, and have the potential to make the biosensor be developed into highly selective devices for a variety of biological analytes [4-5]. However, their instability is a problem for the practical application of phospholipid membranes in biosensor [6-7]. The non-covalent forces responsible for self-organization of the lipid lamellar phase are insufficient to maintain the bilayer structure when, for example, it is exposed to surfactants, organic solvents, or transferred from water into air [8]. Several attempts have been made to overcome it, including hybrid bilayer with a crystalline sheet of S-layer proteins prepared by the Langmuir-Schaefer method [9], cross-linked bilayer prepared by photopolymerization of synthetic lipids [6]. Here a method to fabricate SPB on silicon surface is studied for the biosensor based on imaging ellipsometry. SPB is prepared by covalent immobilization of a supported L- $\alpha$-phosphatidylethanolamine monolayer on silicon surface, and followed by incubation with PEG functionalized phospholipids in aqueous solution. This approach offers better intactness when SPB is exposed to air, even withstood dried under a nitrogen blow, and it may be rehydrated and used for the biosensor.

\section{EXPERIMENT AND DISCUSSION}

\section{A. Experimental section}

Materials: Chemicals used for the buffer (PBS, $\mathrm{pH}$ 7.2; Tris, $\mathrm{pH}$ 7.4; NaAc 4.0) preparation were all of analytical grade. Human immunoglobulin $\mathrm{G}$ (hIgG), goat anti-hIgG serum, fibrinogen (Fib), anti-Fib, Human serum albumin (HSA) and Bovine serum albumin (BSA) were obtained from Sigma. Fetal bovine serum (FBS) was purchased from Hyclone (USA). L- $\alpha$-phosphatidylethanolamine (PE) and Tween 20 were purchased from Sigma-Aldrich. 1,2-Diacylsn-Glycero-3- Phosphoethanolamine-N -[Methoxy(Polyethylene glycol)-2000] (CH3-PEG-PE) and 1,2-Distearoyl-snGlycero-3-Phosphoethanolamine-N-[Carboxyl (Polyethylene Glycol)-2000] (COOH-PEG-PE) were purchased from Avanti Polar Lipids. p-nitrophenylcarbonyl-PEG-1,2dioleoyl-sn-glycero-3- phosphoethanolamine (pNP-PEGDOPE) was synthesized in the laboratory of professor $\mathrm{W}$. Liang[10].

Preparation of proteins and phospholipids solution: For the experiment of non-specific protein adsorption on lipids bilayer, the concentrations of protein solutions were 1 $\mathrm{mg} / \mathrm{ml}$ for h-IgG and Fib, 10\% for anti-hIgG and FBS. For antigen-antibody binding experiment, the concentrations of protein solutions were $0.1 \mathrm{mg} / \mathrm{ml}$ for $\mathrm{hIgG}, 10 \mathrm{mg} / \mathrm{ml}$ for BSA and $10 \%$ for anti-hIgG. PE suspension was prepared at a concentration of $3 \mathrm{mg} / \mathrm{ml}$ in Tris-HCl buffer ( $\mathrm{pH} 7.2$ ) with the addition of $0.5 \%$ Tween $20 . \mathrm{CH}_{3}$-PEG-PE and $\mathrm{COOH}-$ 
PEG-PE were dispersed in Tris-HCl buffer at a concentration of $3 \mathrm{mg} / \mathrm{ml}$ and pNP-PEG-DOPE was dispersed in sodium acetate buffer ( $\mathrm{pH} 4.0$ ) at a concentration of $3 \mathrm{mg} / \mathrm{ml}$.

Contact angle measurement: Water contact angle was measured at room temperature for dried wafers with the sessile drop method. Deionized water $(4 \mu \mathrm{l})$ was gently dropped on surfaces and the contact angle was read directly using a goniometer $\left(\right.$ error $\left.\pm 1^{\circ}\right)$.

Imaging ellipsometry: The experiments were carried out with an imaging ellipsometer developed in our laboratory. As an enhancement of traditional ellipsometry, imaging ellipsometry used a CCD camera to image the ellipsometry response of a larger area sample with lateral distribution in different layer thickness, and the result was grabbed as a digital image and stored in a computer with a grayscale format ( 8 bits, $0-255$ grayscale) for further evaluation by an image-processing program. Once imaging ellipsometer was fixed, the detected intensity "I" ( grayscale ) was the function of the layer thickness (d), namely $I=f(d)$, where $f()$ denoted the function relationship which was determined by layers with known intensity and thicknesses, then the unknown thickness of protein layer could be deduced from the detected intensity according to the function.

Microfluidic system: For the fabrication of SPB and the reaction of SPB with protein, a micro-fluidic system[11] in PDMS was used, which includes an $8 \times 6$ cell array. When the cell array was attached to the silicon slide surface, 48 individual chambers were formed independently and 48 elliptic spots in the array were patterned onto the silicon surface. Each chamber had two access holes where solution could pass in and out of the chamber through them. By such a micro-fluidic system, different molecules were delivered individually to each spot of the array and immobilized on silicon surface simultaneously. Here it was used for patterning SPB or proteins on silicon surface, transporting of sample solution, reaction of molecular and rinsing.

Other preparations: With procedures in Ref.[11], aldehyde groups were introduced on the silicon surface that reacted with amino groups of the proteins or lipids. The thicknesses of protein layers were calibrated by a traditional ellipsometer (SE400, SENTECH, Germany) equipped with a He-Ne laser $(\lambda=632.8 \mathrm{~nm})$. The angle of incidence was $70^{\circ}$.

\section{B. Results and discussion}

Here SPB consisted of two different phospholipids. One was PE covalently immobilized on silicon surface to form a monolayer, another was PEG functionalized PE adsorbed on the monolayer by hydrophobic interaction to form a SPB.

SPB was prepared in the microfluidic system. PE solution was delivered to the silicon surface through the microfluidic system and PE was covalently attached on surface by an imine linkage formed between the aldehyde group on surface and the amine group of PE. After $30 \mathrm{~min}$ incubation, Tris- $\mathrm{HCl}$ buffer was used to rinse the surface, then PEG functionalized PE solution was delivered and incubated with PE monolayers for $30 \mathrm{~min}$ to form SPB. Finally SPB was successively rinsed with Tris- $\mathrm{HCl}$ buffer and purified water.

\section{a. Effect of detergent and the structure of the lipid layer}

The detergent can induce transition from lipid vesicles to lipid-detergent mixed micelles [12], so the molar lipid/detergent ratio (denoted by R) will be an important factor for the forming of the PE layer and the PE-( PEGPEs) bilayer on silicon slide, which is studied in the followings.

The detergent was Tween20 (T). The PE solution $30 \mu \mathrm{l}$ with different R (3:2 $(10 \mathrm{mg} / \mathrm{ml} \mathrm{PE}, 1 \% \mathrm{~T}), 9: 2(30 \mathrm{mg} / \mathrm{ml} \mathrm{PE}$, $1 \% \mathrm{~T}), 15: 2(50 \mathrm{mg} / \mathrm{ml} \mathrm{PE}, 1 \% \mathrm{~T})$ and $30: 1(10 \mathrm{mg} / \mathrm{ml} \mathrm{PE}$, $0.05 \% \mathrm{~T})$ respectively) and the pNP-PEG-DOPE solution $(5 \mathrm{mg} / \mathrm{ml}) 30 \mu \mathrm{l}$ were used to form the lipid layer. The thickness of lipid layer (denoted by d) was measured by the traditional ellipsometer. Experiments were repeated three times and results were presented in Table 1.

The surface area about $1 \mathrm{~mm}^{2}$ of lipid layer prepared by microfluidic system was too small to measure the contact angle, so a similar layer was prepared on a silicon slide $(10 \times 7 \mathrm{~mm})$ dipped in PE solution instead of the microfluidic system. After these slides were incubated in PE solution with different $\mathrm{R}$ over night at room temperature, their contact angles (denoted by $\theta$ ) were measured. As a comparison, contact angles of silicon slides modified by glutaraldehyde were also measured. The experiment was repeated three times and results were presented in Table 1.

From Table 1, that the contact angle of silicon slide modified by glutaraldehyde $(\theta \mathrm{CHO})$ was a constant showed these silicon slides with similar property. With the increase of R, the thickness of PE layer (d1) was gradually increased, but the thickness of layer after pNP-PEG-DOPE across PE layer (d2) almost remains constant, and d1 gradually close to d2. Also, the contact angle of PE layer ( $\theta \mathrm{PE})$ was decreased. Considered that each phospholipid had one hydrophilic head group and two hydrophobic tails, and it

Table 1. Thickness and the contact angle of lipid layers and the silicon slide.

\begin{tabular}{|c|c|c|c|c|}
\hline $\mathrm{R}$ & $\mathrm{d} 1(\mathrm{~nm})$ & $\mathrm{d} 2(\mathrm{~nm})$ & $\theta \mathrm{CHO}\left(^{\circ}\right)$ & $\begin{array}{c}\theta \mathrm{PE} \\
\left(^{\circ}\right)\end{array}$ \\
\hline $3: 2$ & 0.67 & 1.38 & 32.5 & 58.2 \\
\hline $9: 2$ & 1.02 & 1.40 & 32.5 & 48.1 \\
\hline $15: 2$ & 1.39 & 1.44 & 32.5 & 45.7 \\
\hline $30: 1$ & 1.41 & 1.43 & 32.5 & 43.1 \\
\hline
\end{tabular}




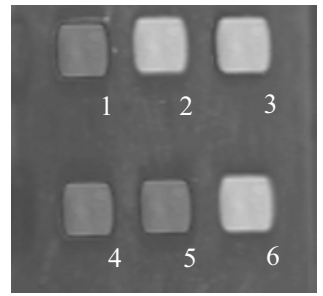

Fig.1. Image in grayscale of complex layers with 6 spots. Spot 1 PE layer, spot 2 PE-hIgG $(\mathrm{pH}=4.0)$, spot 3 PE-hIgG $(\mathrm{pH}=7.2)$, spot 4 PE-(pNP-PEGDOPE) bilayer, spot 5 PE-(pNP-PEG-DOPE)-hIgG (pH=4.0), spot 6 PE(pNP-PEG-DOPE)-hIgG ( $\mathrm{pH}=7.2)$.

was covalently immobilized on silicon slide by its hydrophilic group, so it could be gotten that the PE layer was from monolayer to bilayer with the increase of $\mathrm{R}$.

To judge what the upper layer of bilayer was after pNPPEG-DOPE across PE layer, the adsorption of protein hIgG with different $\mathrm{pH}$ (4.0 and 7.2) was used since -pNP group was inert to $\mathrm{hIgG}$ in buffer with $\mathrm{pH}<5[13]$. For the $\mathrm{PE}$ layer and the layer after pNP-PEG-DOPE across PE layer, two kinds of hIgG solution (prepared by PBS buffer and NaAc buffer to obtain different $\mathrm{pH}$, respectively) were pumped across these layers, respectively. After 10 min's incubation, rinsed with buffer (the same as hIgG solution) and purified water. All experiments mentioned above were done on the same silicon slide with the microfluidic system. After dried with a nitrogen blow, the silicon slide was detected with imaging ellipsometry, and results (relative to $\mathrm{R}=30: 1$ ) were shown in Fig.1. Obvioiusly, the value in grayscale of PE layer (spot 1) was lower than that of PE-hIgG layer (spot 2\&3), which showed hIgG has been absorbed on them. The concentration and volume of phospholipid solution in our experiment were enough [14] to make the lipid layer saturation on silicon slide. It ensured hIgG was absorbed on PE monolayer by hydrophobic interaction (spot 2\&3), but not covalently linked with aldehyde group of silicon slide. For the layer after pNP-PEG-DOPE across PE layer, hIgG with $\mathrm{pH}=7.2$ was adsorbed on it (spot 5), but hIgG with $\mathrm{PH}=4.0$ not (spot 4). Combined that -pNP group was inert to hIgG in buffer with $\mathrm{pH}<5$, it proved that pNP-PEG-DOPE adsorbed on PE monolayer and formed PE-pNP-PEG-DOPE bilayer.

It also found other PEG functionalized PE like $\mathrm{CH} 3$ PEG-PE and COOH-PEG-PE could assemble the similar bilayer, so they were used to study the stability of SPB and the bioactivity of protein on SPB respectively since - $\mathrm{CH} 3$ group was inert to protein and $-\mathrm{COOH}$ group could covalently immobilize protein on the bilayer.

\section{b. Stability of supported phospholipids bilayer}

In practical experiments various buffers were used for biochemical analysis, so the stability of SPB on silicon surface was studied under different buffers. Here, we fabricated a PE-(CH3-PEG-PE) bilayer on silicon surface, and chose the following buffers which were pumped across the SPB for $5 \mathrm{~min}$ : $2 \mathrm{M}$ sodium chloride, $10 \mathrm{mM}$ glycine, 10 $\mathrm{mM}$ sodium carbonate and $100 \mathrm{mM}$ hydrochloric acid. No thickness change of SPB was observed with imaging ellipsometry. Then, these silicon slides were immersed into different protein solutions (IgG, Fib, HSA and FBS) within $10 \mathrm{~min}$. Results showed that almost no protein was adsorbed, which presented the fabricated phospholipids bilayer could withstand various buffers.

Conventional SPB is quickly destroyed upon exposure to air/water interface and must be remained underwater at all time, which makes it not suitable for biosensor applications, such as remote sensing applications that require drying SPB for storage, shipment, and rehydration. To determine whether SPB on silicon surface can meet the requirement or not, we performed multiple cycles of drying and rehydration for SPB. After 5 cycles of drying and rehydration, we placed the silicon slide into FBS solution for $10 \mathrm{~min}$. Results showed that with the increase of drying and rehydration cycles, the adsorption of molecules in FBS also increased, which presented that the drying and rehydration operation might destroy parts of the prepared phospholipids bilayer. However, within one cycle of drying and rehydration, the signal we detected was under noise. For most biosensor, it was common to drying and rehydration only one cycle for storage and shipment, so the lipid bilayer we prepared could meet the requirement.

\section{c. Non-specific adsorption of proteins.}

The ability of SPB on silicon surface to resist nonspecific protein adsorption from the aqueous solutions was determined. The terminal methyl group of CH3-PEG-PE is inert, which can avoid protein attaching covalently on bilayer. Fib, HSA and hIgG solutions were injected across SPB, respectively. After $30 \mathrm{~min}$ incubation, none of the proteins explored caused non-specific adsorption on the bilayer measured with imaging ellipsometry. The adsorption of biomolecules on the bilayer from two more complex solutions, anti-hIgG and FBS, were studied with imaging ellipsometry, also no adsorption occurred on the bilayer. These results were in agreement with that in other work[6], which indicated that SPB was quite resistant to nonspecific protein adsorption.

\section{d. Antigen - Antibody Binding.}

$\mathrm{hIgG} /$ anti-hIgG binding was employed as a model of antigen - antibody interaction on SPB (the first layer was also PE monolayer and the second layer was COOH-PEGPEs). hIgG solution was pumped across the bilayer, and then hIgG would be immobilized covalently on surface by stable carbamate bond formed between carboxyl group of 
PEG chain's end and amino group of hIgG. After incubation for $30 \mathrm{~min}$, BSA solution was injected across the bilayer for 30 min. For comparison, hIgG was immobilized covalently on the silicon surface with aldehyde groups directly. Furthermore $10 \%$ anti-hIgG in PBS buffer was pumped across hIgG layers for $30 \mathrm{~min}$ and rinsed with PBS buffer and purified water. All experiments mentioned above were done on one single silicon slide with the microfluidic system. After dried with a nitrogen blow, the silicon slide was measured with imaging ellipsometry and the result was shown in Fig. 2

The thickness of SPB-hIgG layer $(4.4 \mathrm{~nm})$ was lower than that of hIgG layer $(5.0 \mathrm{~nm})$ on silicon slide, but more anti-hIgG $(5.7 \mathrm{~nm})$ bound on SPB-hIgG layer than that (4.6 $\mathrm{nm})$ on silicon slide. We repeated the experiment many times and almost same results were obtained, which showed that $\mathrm{hIgG}$ molecule attached on phospholipids bilayer can maintain higher bioactivity.

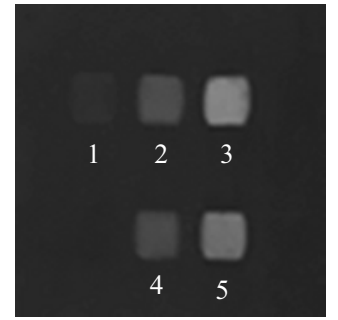

A

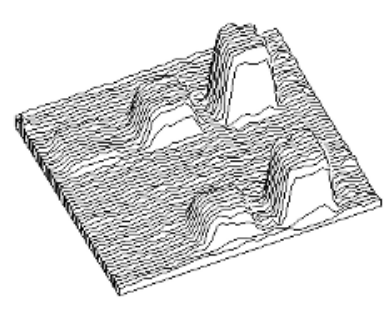

B
Fig.2. Silicon slide with 5 spots patterned different layers shown in grayscale image (A) and the corresponding thickness distribution image (B). Spot 1 SPB layer, spot 2 SPB-hIgG layer, spot $3 \mathrm{SPB}-\mathrm{hIgG} /$ anti-hIgG complex layer, spot $4 \mathrm{hIgG}$ layer, and spot $5 \mathrm{hIgG} /$ anti-hIgG complex layer.

\section{Conclusions}

SPB was fabricated on silicon surface for biosensor based on imaging ellipsometry, which was highly protein resistant and stable, and could withstand multiple cycles of drying and rehydration, remaining intact in various buffers. Moreover, proteins could be immobilized covalently on the bilayer by the terminal carboxyl group of PEG chain's end, which its bioactivity was higher than that immobilized on silicon surface directly. It showed the biosensor with the potential to be further developed into a sensitive immunoassay technique.

\section{ACKNOWLEDGMENT}

The national natural science foundation of China (20845003) and Chinese Academy of Sciences
((KJCX2.YW.M02 and M04)) are acknowledged for their supports.

\section{REFERENCES}

1. Jin G, Tengvall P, Lundstrom I et al, (1995), A biosensor concept based on imaging ellipsometry for visualization of biomolecular interactions. Biochem.232:69-72.

2. Jin G, Jansson R, Arwin H,(1996) Visualization of thin transparent organic films with imaging ellipsometry. Rev. Sci. Instrum. 67:29302936.

3. Kanda V, Kariuki J.K, Harrison D.J et al, (2004), Label-free reading of microarray-based immunoassays with surface plasmon resonance imaging.Anal. Chem. 76:7257-7262.

4. Groves, J.T.; Boxer, S.G. (2002) Micropattern formation in supported lipid membranes, Acc Chem Res. 35:149-157.

5. Yang T.L, Jung S.Y, Mao H.B et al, (2001) Fabrication of phospholipid bilayer- coated microchannels for on-chip immunoassays Anal Chem. 73:165-169.

6. Ross E.E, Rozanski L.J, Spratt T et al,(2003) Planar Supported Lipid Bilayer Polymers Formed by Vesicle Fusion. 1. Influence of Diene Monomer Structure and Polymerization Method on Film Properties Langmuir. 19:1752-1765.

7. Phillips S.K, Dong Y, Carter D et al, (2005), Stable and Fluid Ethylphosphocholine Membranes in a Poly(dimethylsiloxane) Microsensor for Toxin Detection in Flooded Waters. Anal. Chem. 77: 2960-2965.

8. McBee T. W, Saavedra S.S, (2005),Stability of Lipid Films Formed on $\gamma$-Aminopropyl Monolayers, Langmuir, 21:3396-3399.

9. Wetzer, B., Pum, D., Sleytr, U.B.,(1997),S-layer stabilized solid supported lipid bilayers, J. Struct. Biol. 119:123-128.

10. Liang W., Levchenko T., Khaw B.A. et al,(2004),ATP-containing immunoliposomes specific for cardiac myosin, Curr. Drug Deliv. 1: 1-7.

11. Wang Z.H, Meng Y.H, Ying P.Q et al, (2006), A label-free protein microfluidic array for parallel immunoassays. Electrophoresis, 27: 4078-85.

12. Lasch J, (1995), Interaction of detergents with lipid vesicles, Biochemica et Biochimica Acta 1241: 269-292.

13. Torchilin V.P, Levchenko T.S, Lukyanov, ( 2001), pNitrophenylcarbonyl-PEG-PE-liposomes: fast and simple attachment of specific ligands, including monoclonal antibodies, to distal ends of PEG chains via p-nitrophenylcarbonyl groups, Biochimica et Biophysica Acta 151:397-411.

14. Keller C.A, Kasemo, (1998), Surface Specific Kinetics of Lipid Vesicle Adsorption Measured with a Quartz Crystal Microbalance. B. Biophys. J. 75: 1397-1402.

Corresponding author: G. Jin

$\begin{array}{ll}\text { Institute: } & \text { Institute of Mechanics, } \\ \text { Street: } & \text { Bei-si-huan west road } \\ \text { City: } & \text { Beijing } 100190 \\ \text { Country: } & \text { China } \\ \text { Email: } & \text { gajin@imech.ac.cn }\end{array}$

Co-corresponding author: W.R. Chang

Institute: Institute of Biophysics

Street: 15 Datun road, Chaoyang district

City: $\quad$ Beijing

Country: China

Email: $\quad$ wrchang@sun5.ibp.ac.cn 